\title{
Erratum to: Heregulin-1ß and HER3 in hepatocellular carcinoma: status and regulation by insulin
}

Corina Buta ${ }^{1}$, Eva Benabou' ${ }^{1}$, Marie Lequoy ${ }^{1,2}$, Hélène Régnault ${ }^{1,3}$, Dominique Wendum ${ }^{1,4}$, Fatiha Merabtene ${ }^{1,5}$, Hamza Chettouh ${ }^{1}$, Lynda Aoudjehane ${ }^{1,6}$, Filomena Conti ${ }^{1,6,7}$, Yves Chrétien ${ }^{1}$, Olivier Scatton 1,7, Olivier Rosmorduc ${ }^{1,3}$, Françoise Praz ${ }^{1}$, Laetitia Fartoux ${ }^{1,3}$ and Christèle Desbois-Mouthon ${ }^{1 *}$

\section{Erratum description}

In the original publication of this article [1], one of the co-authors' names contained a spelling error. 'Fatiha Meratbene' should therefore have been listed as 'Fatiha Merabtene'. The original publication has now been modified as well to reflect these changes.

\begin{abstract}
Author details
'Sorbonne Universités, UPMC Univ Paris 06, INSERM, Saint-Antoine Research Center, 34 rue Crozatier, F-75012 Paris, France. ${ }^{2}$ Department of Hepatology, AP-HP, Saint-Antoine Hospital, F-75012 Paris, France. ${ }^{3}$ Department of Hepatology, AP-HP, Pitié-Salpétrière Hospital, F-75013 Paris, France. ${ }^{4}$ Department of Pathology, AP-HP, Saint-Antoine Hospital, F-75012 Paris, France. ${ }^{5}$ Histomorphology Platform, UMS 30 Lumic, F-75012 Paris, France. ${ }^{6}$ Human HepCell, Saint-Antoine Hospital, F-75012 Paris, France. ${ }^{7}$ Department of Hepatobiliary Surgery and Liver Transplantation, AP-HP, Pitié-Salpétrière Hospital, F-75013 Paris, France.
\end{abstract}

Received: 7 September 2016 Accepted: 22 September 2016 Published online: 28 September 2016

\section{References}

1. Buta $C$, et al. Heregulin-1B and HER3 in hepatocellular carcinoma: status and regulation by insulin. J Exp Clin Cancer Res. 2016;35:126. doi:10.1186/s13046016-0402-3.

\footnotetext{
* Correspondence: christele.desbois-mouthon@inserm.fr

'Sorbonne Universités, UPMC Univ Paris 06, INSERM, Saint-Antoine Research
} Center, 34 rue Crozatier, F-75012 Paris, France 